\title{
APPLICATIONS, LIMITATIONS, AND IMPROVEMENTS IN VISIBLE LIGHT COMMUNICATION SYSTEMS
}

\author{
FAZLULLAH KHAN $^{1}$, FARMAN KHAN ${ }^{2}$, QAMAR JABEEN ${ }^{1}$, SYED ROOHULLAH JAN $^{1}$, SHAHZAD KHAN $^{1}$ \\ ${ }^{1}$ Department of Computer Science, Abdul Wali Khan University Mardan, Pakistan \\ ${ }^{2}$ Department of Computer Science, Bacha Khan University Charsadda, Pakistan \\ EMAIL: fazlullah@awkum.edu.pk,
}

Revised March 2016

ABSTRACT. Wireless communication networks using unlicensed frequency band faces certain challenges like unrestrained interfering and bad quality of transmission. To surmount the scarcity of frequency band, a new technique for wireless communication is compulsory to adapt the exponentially rising wireless communication demand. Visible light communication systems (VLCN) offer a replacement to the existing standards of wireless communication, through light from light-emitting diodes (LEDs) as the mean of communication. As LEDs twinkle repeatedly at a high speed such that human eye cannot perceive changes in light intensity, but a perceptive photodiode detect the on-off attitude and can interpret the data implanted within the light. This paper investigates different issues in the existing wireless communication networks, and studies how VLCN can resolve these issues, and proposes design of the VLCN. Moreover, applications, solution to current issues and future improvements are discussed in this paper.

Keywords: Visible light communication system; 802.15.7 and LED communication; free-space line-of-sight communication; LED-to-LED communication.

1. Introduction. Visible light communication (VLC) networks use LEDs as senders and receivers. VLC offers a new mechanism to make a low-bit-rate (LBR) wireless adhoc communication system for short range communication [1]. LED-to-LED (L2L) VLC networks are made by connecting VLCN devices on adhoc basis for short range communication on free-space optical line-of-sight medium. Less complex VLC adhoc networks are beneficial in establishing local area networks (LANs) including smart home and illumination, sensor and home networks, etc or in connecting consumer electronic devices. These networks are capable of adding and removing end nodes on the go and are economical. In these networks end nodes do not work with photo-detectors to obtain information rather LEDs are used for receiving information [2]. In VLCN 8-bit microcontroller is used to run adhoc communication and to manage the network and medium access protocols.

There are numerous advantages of VLC, as it is visible compare to radio communication, so all the senders and receivers of a message are seen (security). Moreover, VLC does not employ electromagnetic-waves and therefore have no side effect on human especially pregnant women (health). Similarly, VLC does not make use of radio frequency band and therefore is very helpful for limited bandwidth applications (economical). Light is present everywhere, so we can mingle VLC with lighting design to allow VLCN to work together with the lighting systems glowing in many houses, workplaces, schools, colleges, and Universities (opportunistic). The use of radio frequency inside aircrafts particularly during take-off and landing is very dangerous and can cause huge losses including human lives. The use of VLCN on aircraft is a better option to utilize which can reduce aircraft crash incidents (safety).

As communal reliance upon wireless communication networks is rapidly increasing, wireless technology has 
UNITED

STATES

FREQUENCY

ALLOCATIONS

\section{THE RADIO SPECTRUM}

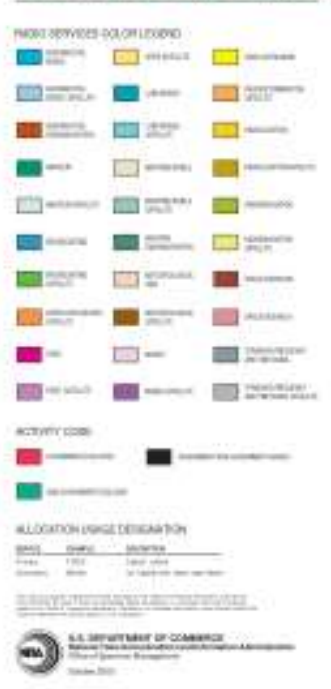

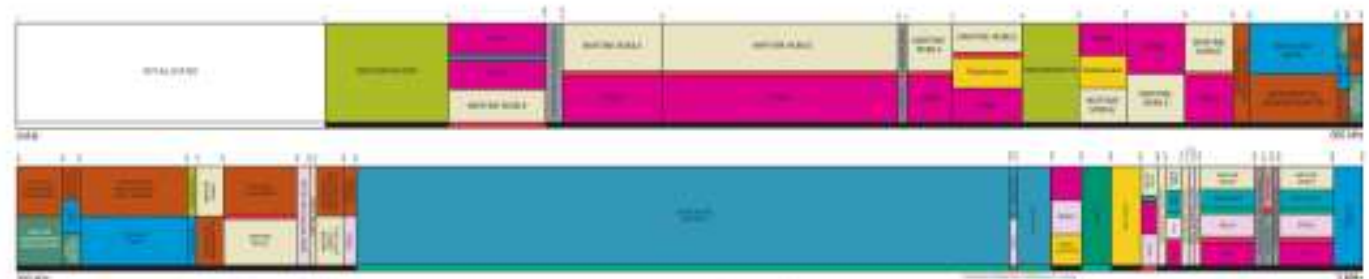
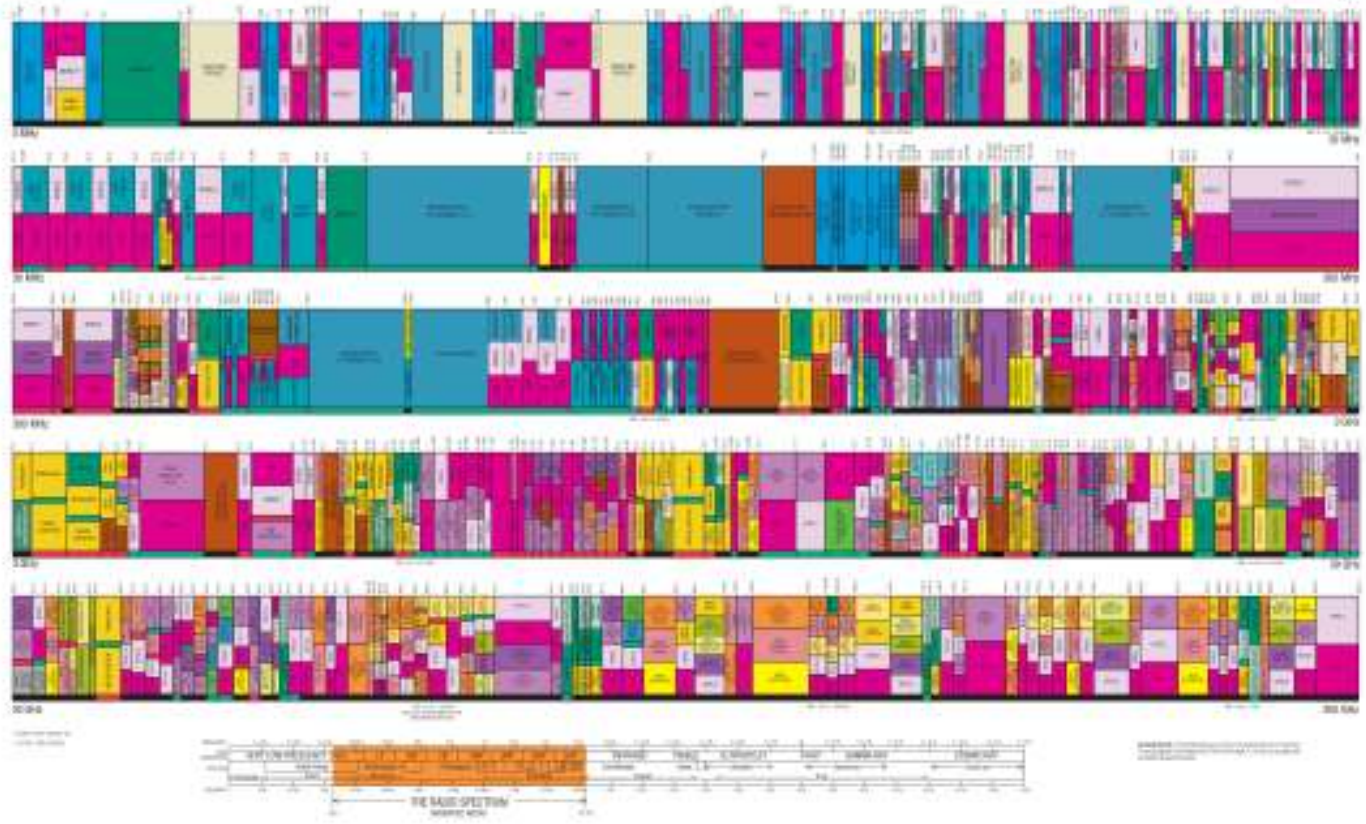

Figure-1. US Frequency Allocations

to cope with the growing demand of the users. Mobile phones, personal digital assistants, global positioning systems, palmtops, laptops, cellular networks, etc transfer information using frequency spectrum. Due to the spectrum scarcity new wireless technology are not safe to implement in the current spectrum and this force us to explore new and alternate wireless technologies for even expansion of new wireless communication systems.

The Federal Communication Commission (FCC) is the regulatory authority of wireless communication in the US [3]. A frequency band has been allocated to each communication company and has to efficiently utilize the allocated band. The crowding of the spectrum is evident from Figure. 1 [4], and still the demand is growing. The projected calculation that US have spectrum deficiency in 2013 is depicted in Figure. 2. Therefore, an efficient utilization of the spectrum is the cry of the day.

In addition to the crowding of the spectrum, uncontrolled interference and bad quality of transmission is also an issue in current wireless communication networks. Concurrent usage of frequency spectrum causes interference because of electromagnetic phenomenon used by wireless devices that could result delay or loss of information. For example, use of electronics devices during take-off and landing of air planes have direct concern to human lives. The Federal Aviation Administration (FAA) says that these electronic devices create interference to the aircraft's navigation and communication systems, while the FCC claims that mobile devices used on aircrafts will disturb cellular towers on the ground. Some studies shows that electronic devices on airplane can surpass allowable emanation level for safety with respect to some avionics [5]. It is evident that in certain environments using frequency band is not practicable where safety, data integrity, and accuracy are highly important. Therefore, in such environment the need of VLCN is utmost important. 


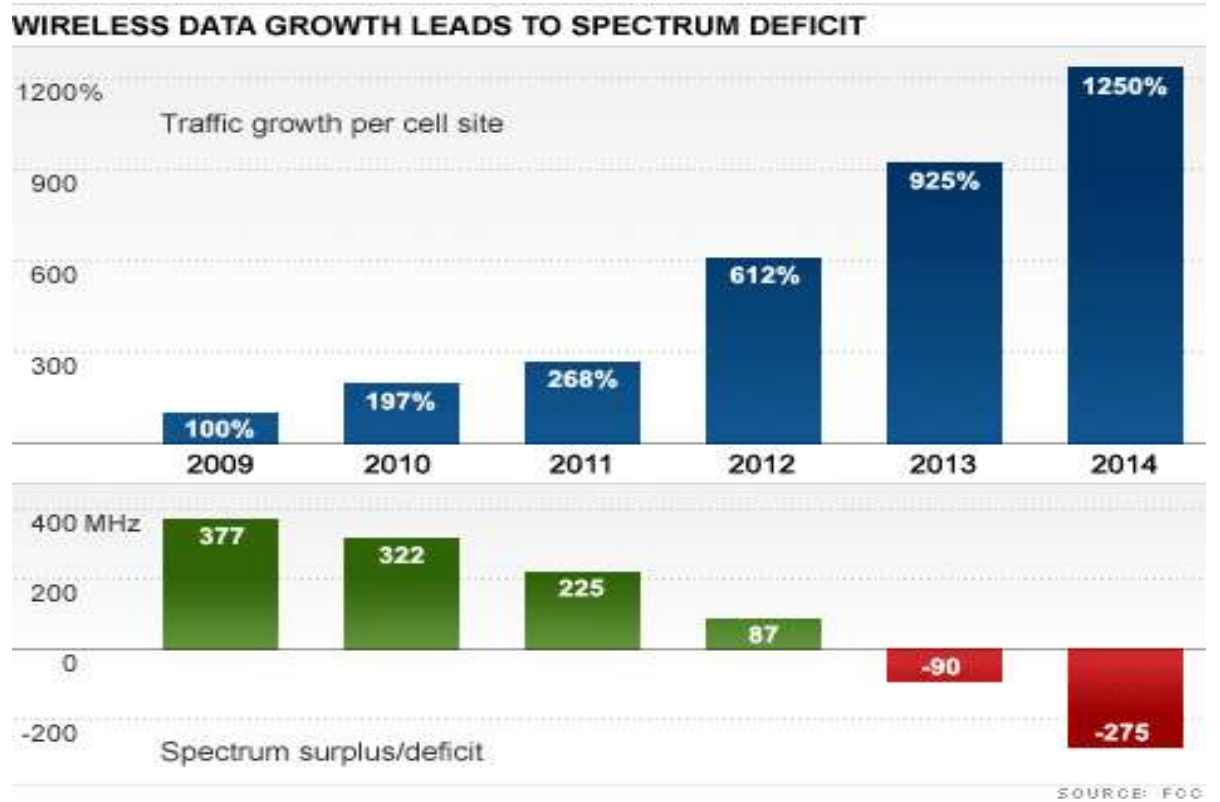

Figure-2. Wireless Data Growth Predictions

The rest of the paper is according to the following pattern. Section II reviews the Related Work; Section III describes details of the Limitations and Failure Analysis. Potential applications and design are discussed in Section IV; Section V gives details of the Solutions and Future Improvements, and last Section concludes the paper.

2. Related Work. A good survey on VLCN and free-space optical line-of-sight can be found in [6,7,29]. The practicality of VLCN for customer products and toys is studied in [2, 7]. The VLC Consortium publish regulations of the VLC technology in 2011 [9], whereas VLC IEEE 802.15.7 standard specifies physical and medium access control protocol stack [9]. In [10], the later is responsible for star and peer-to-peer networks, and the former consist of three different modulation schemes. For better throughput and high speed communication efforts on new regulations are available in [10]. The standard also describes data encryption, communication, authentication, and sharing of data across the 802 architecture. Similarly, there are few alternating technologies to wireless communication systems, i.e. cognitive radio networks, and laser communication systems.

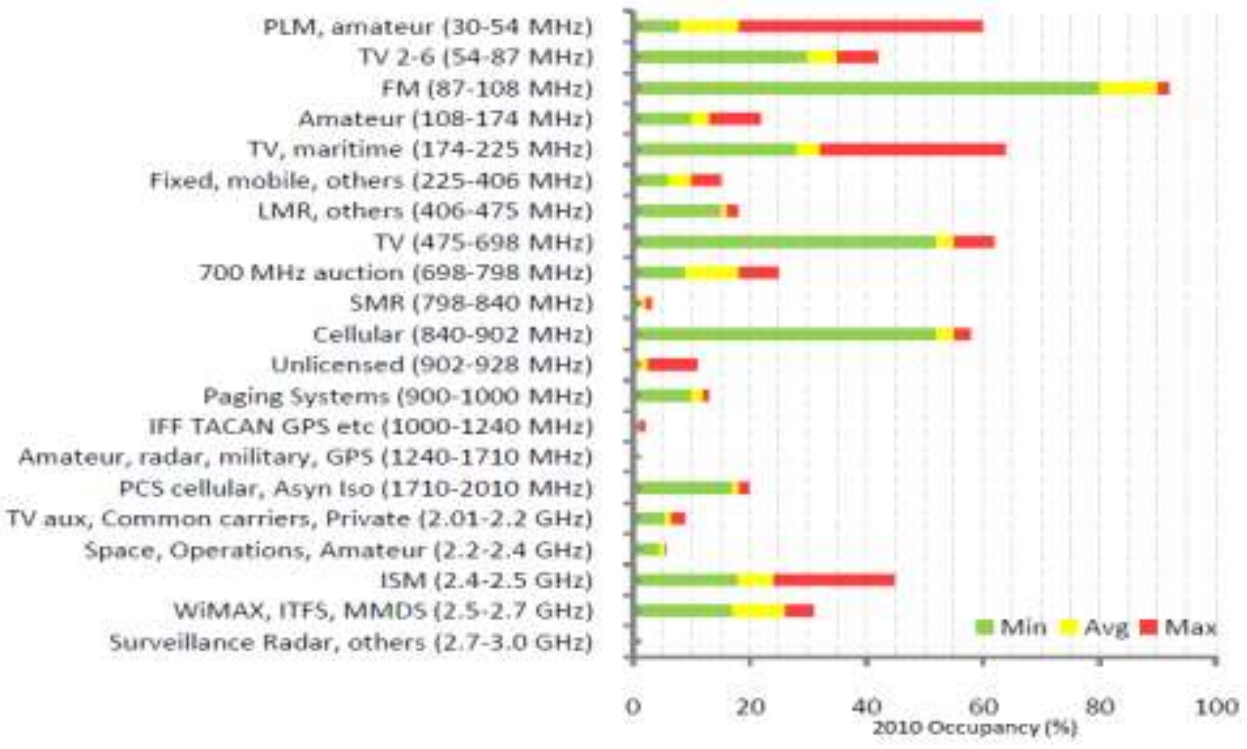

Figure-3. Estimated occupancy for 2010 
Cognitive Radio Networks (CRN). Cognitive Radio (CR) is a promising technology for solving the challenges posed by spectrum scarcity. The CR node uses the licensed spectrum when it the spectrum is idle without interfering the Primary User (PU). In CRN terminology, the PU has high precedence to access the spectrum as PU pays for the spectrum. The CR node also called Secondary User (SU) has low precedence as SU does not pay for using the vacant spectrum. In CRN networks, SU must not create any harmful interference to PU. When a PU arrives, SU should immediately vacate the current channel and move to another available channel. The spectrum utilization in the US was studies by a group of researchers at the Illinois Institute of Technology in Chicago, IL, and observed the FCC allocated spectrum for three years in 2010; their results regarding spectrum utilization is shown in Figure. 3. They obtained spectrum occupancy by measuring spectral density against a pre-defined threshold. Our work on cognitive radio networks and cognitive radio sensor networks can be found in [13, 14, 15,16, 17, $18,19,20,21,22]$.

Laser Communication Systems (LCS). LCS performs wireless communication, transferring data in free-space by bombarding a laser beam. These systems are effective as they are not overcrowding the spectrum and are not regulated by Governments. Like radio communication these systems do not have overhead of processing different regulations rules and are fast enough. These systems are secure because eavesdropping interrupts the transmission; use low power; communicate at 1.25G Gps upto a distance of $6 \mathrm{Km}$. However, they need line-of-sight path, where sender receiver pair cannot not be mobile. Communication is successful when the path is tuned accurately, and the antenna of sender receiver is angled precisely. However, it is dangerous when living beings are intensively exposed to laser beam [11,12].

3. Limitations in the Existing work. There are numerous issues and limitations in the design and implementation of the VLCN ranging from analog to digital transmission as stated below.

Ambient Lighting Source. The presence of a stronger light other than our system light generate low signal-to-noise ratio (SNR), which causes distortion in data. This results in no or inaccurate information transfer.

Line-of-sight Propagation. The sender receiver pair must be in the line-of-sight with each other; otherwise there will be no communication, and even if the sender receiver angle is altered very slightly, the communication range and accuracy will be reduces considerably.

Digital Issues. It is difficult to find the precise sampling rate of the ADC. The output from the ADC is not constant. The CCS and IDE have issues in compiling program, where errors are not properly shown, and many times restarting the IDE is required.

Analog Issues. We have two types of analog issues as below;

LED Brightness. Original LEDs are very dim and can perform very short range communication only. A power MOSFET is required for transmitting strong signal to the LED during transmission.

MOSFET Limitations. MOSFET devices produce more heat and that could make the system fail, therefore, MOSFET is not a good choice to design with.

4. Potential Applications and Design. Light is glowing ubiquitously, opening many ways to VLCN. There are different areas where VLCN can offer communication services without interfering and overcrowding the radio spectrum. Such area includes but not limited to traffic lights, television screens, and hospitals etc.

Traffic Lights. Upon red in traffic signals, pedestrian and drivers are idle and this kills their precious time during travelling. The VLCN provides an opportunity to connect the travelers to the stop/traffic light through their mobile phones or car head light for obtaining some kind of information. This system provides another means of accessing information free of cost.

Television Set. Unlike a stop light, hundreds of pixels that are continuously glowing in television set to project an image to the audiences. Among the many LEDs it is possible to dedicate a few to communication via VLCN. This system will ease the user in getting information of what is broadcasting on other channels without toggling the current channel and internet connection. 


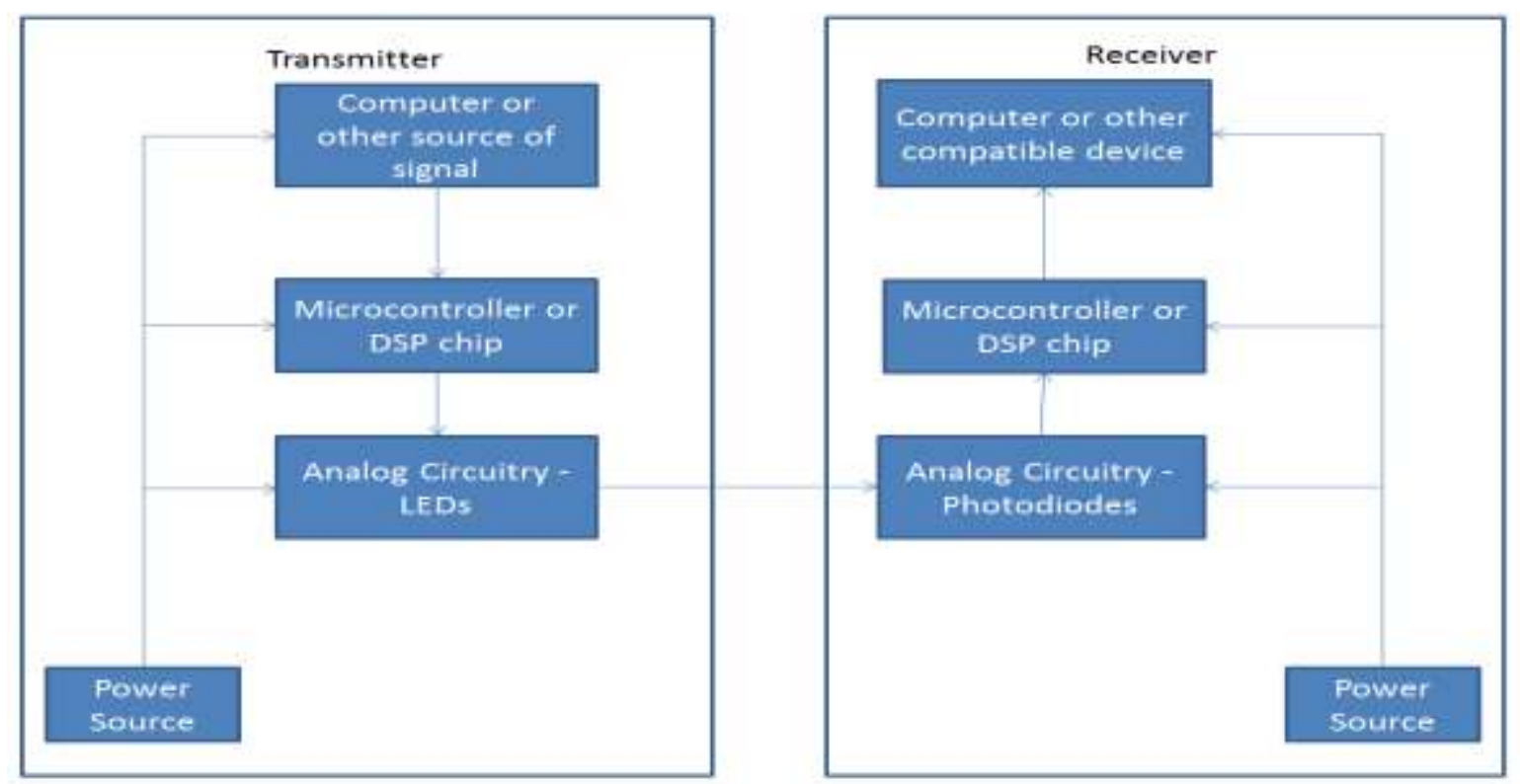

Figure-4. Functional Block Diagram

Hospitals. There are thousands reasons to adopt wireless technology in hospitals. For example, updating and monitoring patient records, viewing health condition and medical images of patients. Accuracy and in-time delivery of information in health-care systems is the main concern. Other important concerns are the data confidentiality of patients, and interference from wireless devices may cause medical devices to shutdown or produce inaccurate results. The VLCN do not permit mobility and cannot communicate through barriers, hence making communication secure. The VLCN is a better choice to be installed at hospitals, because it does not use Industrial Scientific and Medical band and will not be interfering with medical devices. The functional block diagram of our proposed system in drawn in Figure. 4, whereas application and their requirements are discussed in Table I. The five parameters for each application are shown column wise. The first column represents the bandwidth utilization for each application. The required bandwidth can be low, always low, sometimes low and sometimes high, or always high. The second column entries are the minimum allowable delay in information transmission. The middle column states effects of data loss is critical, not critical, or moderate. The fourth column shows reliability requirements of the system. In other words how much we can rely on the system, very high, high, or normal. The final column discusses which security features should be necessary for a partial application, like data integrity, eavesdropping, authentication, and confidentiality of data.

Table 1. Applications and their Requirements

\begin{tabular}{|c|c|c|c|c|c|}
\hline \multirow{2}{*}{ Application } & \multicolumn{5}{|c|}{ Requirements } \\
\cline { 2 - 6 } & Bandwidth & Delay & Data Loss & Reliability & Security \\
\hline Remote Control Apps & Low & $5 \mathrm{sec}$ & Critical & Very high & Integrity \\
\hline Real-time critical apps & Always low & $\begin{array}{c}3000 \\
\mathrm{msec}\end{array}$ & Critical & Very high & $\begin{array}{c}\text { authentication } \\
\text { confidentiality }\end{array}$ \\
\hline Real-time non-critical apps & Low to high & $\begin{array}{c}250 \\
\mathrm{msec}\end{array}$ & Moderate & High & $\begin{array}{c}\text { authentication } \\
\text { confidentiality }\end{array}$ \\
\hline Office/ Medical IT & High & $1 \mathrm{sec}$ & Not critical & Normal & $\begin{array}{c}\text { integrity, } \\
\text { authentication } \\
\text { confidentiality }\end{array}$ \\
\hline
\end{tabular}


5. Solutions and Future Improvements. VLCN has numerous advantages over other wireless communication systems, e.g. its high bandwidth ranging from $430-750 \mathrm{THz}$, which is very large than radio band from $3 \mathrm{KHz}-$ $300 \mathrm{GHz}$ [3]. VLCN will provide high bandwidth to a large number of users, and light is everywhere so it is abundance. This system is safe and may be installed in aircraft for intra-communication among the crew and passengers. This system is secure as all sender receivers are visible to each other, and broadcasting from other rooms/external sources is not possible.

Some modifications and improvements to the issues in VLCN are suggested here. For example, to overcome the issues of ambient lightning source, SNR will be increased based on the voltage signal produces by ambient lightning source in combination with sender signal.

Similarly, the VLCN throughput will be increased if the LEDs are communicating with no hindrance to sensors. The sender receiver angle must be accurately and precisely adjusted, so that they are in the line-of-sight with each other. Some future improvements are discussed here.

Digital Improvements. Digital improvement mainly refers to hardware improvements like processor, FPGAs, and other digital signal processing chips.

Field-Programmable Gate Array (FPGA). FPGA can perform concurrent execution, which helps ADC to sample data without interrupting other processes. The same data could be converted to ASCII text by another process. FPGA also handles high frequency signals efficiently, this gives higher transmission rate. A microcontroller performs sequential execution; this why FPGA a better choice to use in VLCN.

Better Processing Chips. If a microprocessor is the choice for future VLCN, some aspects must be bear in mind such as ADCs sampling rate, availability of sampling code, memory and processing speed.

Computer Interface. One of the important parts of VLCN is its interface. Processor with low memory could transfer data for high speed processing to computer through its interface. An alternate is to use the USB UART interface between the microcontroller and computer for transferring data including graphics, text, and videos.

Analog Improvements. In analog improvement we mainly have to focus on the brightness of LED with even low voltage. The LED must be glowing intensively so that long range communication is maintained. Similarly the use of MOSFET may be depleted or improvements to the current design of MOSFET are required to remove the heat issues in it.

6. Conclusion. In VLC network the LEDs are used as a sender and receiver, which make this network an energy efficient, that is why it opens a new direction and opportunity in mobile communication. It has many applications in smart homes, entertainment industry, and medical field with low-cost because light is an essential component of our daily lives. The VLC devices use approximately same amount of energy in all states, i.e. sending, receiving, and idle mode. VLCN is a new system for making secure adhoc networks of low data rate by connecting consumer electronic devices because LED-based networks conceal data and information in light. The VLCN might attain better performances in near future by combine smart illumination with low-cost adhoc networks, and become an aspirant technology for the Internet of Things [23,24,25,26,27, 28,29].

\section{REFERENCES}

[1] Schmid, S., Corbellini, G., S. Mangold, \& T. Gross. (2012). An LED to LED Visible Light Communication System with software-based synchronization. IEEE Optical Wireless Communication Globecom Workshops, pp. 1264-1268.

[2] Tippenhauer, N. O., Giustiniano, D. \& S. Mangold, (2012). Toys Communicating with LEDs: Enabling Toy Cars Interaction. IEEE Consumer Communications and Networking Conference, pp. 48-49.

[3] http://www.fcc.gov/what-we-do viewed 31-3-2016.

[4] https://www.ntia.doc.gov/files/ntia/publications/2003-allochrt.pdf

[5] http://en.wikipedia.org/wiki/Mobile_phones_on_aircraft viewed 31-3-16.

[6] Elgala, H., Mesleh. R., \& Haas, H., (2011). Indoor optical wireless communication: Potential and state-of-the-art. IEEE Communications Magazine on Optical Communications Series, vol. 49, no. 9, pp. $56-62$.

[7] Elgala, H. (2009). Indoor broadcasting via white LEDs and OFDM, IEEE Transaction on Consumer Electronics, vol. 55, no. 3, pp. $1127-1134$.

[8] Schmid, S., Gorlatova, M., Giustiniano, D., Vukadinovic, V., \& Mangold, S., (2011). Networking Smart Toys with Wireless ToyBridge and ToyTalk, in the Poster Session at INFOCOM.

[9] "Visible Light Communications Consortium," http://www.vlcc.net viewed 31-3-2016. 
[10] 802.15.7, (2011). Short-Range Wireless Optical Communication using Visible Light, IEEE standard for local and metropolitan area networks, Part 15.7:

[11] "Laser Communication" http://lasercommunications.weebly.com/ viewed 31-3-2016.

[12] Omega, (2016). the Home Gigabit Access project," http://www.ict-omega.eu/ viewed 31-3-16.

[13] Khan, F., \& Bashir, F. (2012). Dual Head Clustering Scheme in Wireless Sensor Networks. in the IEEE International Conference on Emerging Technologies (pp. 1-8). Islamabad: IEEE Islamabad.0

[14] Jan, M. A., Nanda, P., He, X., \& Liu, R. P. (2013). Enhancing lifetime and quality of data in cluster-based hierarchical routing protocol for wireless sensor network. High Performance Computing and Communications \& 2013 IEEE International Conference on Embedded and Ubiquitous Computing (HPCC_EUC), 2013 IEEE 10th International Conference on (pp. 1400-1407). IEEE

[15] Jan, M.A., Nanda, P., \& He, X. (2013). Energy Evaluation Model for an Improved Centralized Clustering Hierarchical Algorithm in WSN in Wired/Wireless Internet Communication, Lecture Notes in Computer Science. (pp. 154-167), Springer, Berlin, Germany.

[16] Khan, F., Nakagwa, K.. (2013). Comparative Study of Spectrum Sensing Techniques in Cognitive Radio Networks. In IEEE World Congress on Communication and Information Technologies (p. 8). Tunisia: IEEE Tunisia

[17] Jan, M.A., \& Khan, M. (2013). A Survey of Cluster-based Hierarchical Routing Protocols. IRACST-International Journal of Computer Networks and Wireless Communications (IJCNWC). Vol.3, 138-143.

[18] Khan, F., \& Nakagwa., K. (2012). Cooperative Spectrum Sensing Techniques in Cognitive Radio Networks. in the Institute of Electronics, Information and Communication Engineers (IEICE), Japan , Vol -1, 2.

[19] Jan, M.A., \& Khan, M. (2013). "Denial of Service Attacks and Their Countermeasures in WSN". IRACST-International Journal of Computer Networks and Wireless Communications (IJCNWC). Vol.3.

[20] Khan, F., \& Nakagwa, K. (2012). Performance Improvement in Cognitive Radio Sensor Networks. in the Institute of Electronics, Information and Communication Engineers (IEICE) , 8.

[21] Jan, M.A., Nanda, P., He, X., \& Liu, R. P. (2014). "PASCCC: Priority-based application-specific congestion control clustering protocol". Computer Networks, vol. 74, 92-102

[22] Khan, F., \& Kamal, S. A. (2013). Fairness Improvement in long-chain Multi-hop Wireless Adhoc Networks. International Conference on Connected Vehicles \& Expo (pp. 1-8). Las Vegus: IEEE Las Vegus, USA.

[23] Khan. F. (2014). Throughput \& Fairness Improvement in Mobile Ad hoc Networks. the 27th Annual Canadian Conference on Electrical and Computer Engineering (p. 6). Toronto, Canada: IEEE Toronto.

[24] Jan, M.A., Nanda, P., He, X., \& Liu, R. P. (2015). "A Sybil Attack Detection Scheme for a Centralized Clustering-based Hierarchical Network," in Trustcom/BigDataSE/ISPA, vol.1, PP-318-325, IEEE.

[25] Jan, M.A., Nanda, P., He, X., \& Liu, R. P. (2014)., "A robust authentication scheme for observing resources in the internet of things environment" in 13th International Conference on Trust, Security and Privacy in Computing and Communications (TrustCom), pp. 205-211, IEEE

[26] Khan, S., \& Khan, F.. (2015). Delay and Throughput Improvement in Wireless Sensor and Actor Networks. $5^{\text {th }}$ National Symposium on Information Technology: Towards New Smart World (NSITNSW) (pp. 1-8). Riyadh: IEEE Riyad Chapter.

[27] Jan, M. A. (2016). Energy-efficient routing and secure communication in wireless sensor networks (Doctoral dissertation), http://hdl.handle.net/10453/43497

[28] Khan, F. (2014). Secure Communication and Routing Architecture in Wireless Sensor Networks. the $3^{\text {rd }}$ Global Conference on Consumer Electronics (GCCE) (p. 4). Tokyo, Japan: IEEE Tokyo.

[29] Khan, S., Khan, F., Arif, F., Jabeen, Q., Jan, M.A., \& Khan, S.A. (2016). Performance Improvement in Wireless Sensor and Actor Networks. In the Journal of Biological and Applied Sciences. 